\title{
8 \\ Icaros, Alice and the OSF DME
}

\author{
J. Scott Marcus \\ BBN Internet Services Corp. \\ 150 Cambridge Park Drive, Room 20/321 \\ Cambridge, MA 02140, U.S.A. \\ (617) 873-3075 phone, (617) 873-5620 FAX \\ smarcus@bbn.com
}

\begin{abstract}
In 1990, the Open Software Foundation (OSF) set out an ambitious quest: to create an integrated management platform that would unify systems management and network management within the context of an object-oriented management framework. The totality of this task proved to be over-ambitious. This paper explores a few of the factors that made the project more difficult than had been assumed.
\end{abstract}

\section{Introduction}

\begin{abstract}
"Icaros was delighted. He flew steadily at first, but when they had got clear out of sight of the haven, he became excited... higher and higher he went, until he came too close to the sun, and the sun melted the wax, and the feathers fell out of his wings, and he dropped into the sea like a stone."
\end{abstract}

W.H.D. Rouse, Gods, Heroes, and Men of Ancient Greece

The disparity between the management technology we would like to have, and the technology that we are capable of successfully delivering today, is daunting. Small wonder, then, that so many network and system management development projects fail!

When the Open Software Foundation (OSF) set out to create a Distributed Management Environment (DME), they did so with high hopes -- and indeed, why should they not have? The OSF represented the technical and marketing might of some of the greatest computer companies in the world. The task before them was the most ambitious that OSF had attempted, but also the most urgently needed: a stable, widely deployed platform that would offer modern software development tools and standards-compliant Application Programming Interfaces (APIs) for both system management and network management. The objectives seemed to many to be challenging, but achievable. The DME was to "unify the worlds of systems and network management" by ushering in a new era of object-oriented distributed management [OSF91]. 
The OSF has been unable to deliver on these promises. They have delivered OSF software, and this software may in time achieve wide deployment [OSF94]; however, the OSF as delivered is a wan shadow of the comprehensive, integrated functionality that was initially intended. The first DME software that the OSF delivered, under the moniker DME 1.0, consisted of the distributed applications that were originally intended to serve as a mere proof of concept for the DME management framework. The conventional network management portions of the DME, the Network Management Option (NMO), do not appear to represent a major advance over the technology that was available to the OSF when it began the DME acquisition process in August of 1990 [OSFRFT]. The Object Management Framework (OMF) with its integral Object Request Broker (ORB), the heart of the modern object-oriented portions of the DME, has been delayed to the point where it was no longer necessary or beneficial for OSF to deliver its own ORB. Perhaps most significant, the vaunted integration of system and network management is still simply nowhere in sight.

The trade press has been quick to fasten on simplistic explanations for this failure, ranging from Machiavellian machinations on the part of OSF's sponsors to gross incompetence on the part of OSF. The reality is, of course, more complex.

It is not the author's intent to cast stones. I have driven my share of management projects into the ground, and am convinced that there is more that we can learn from our failures than from our successes. The DME represents an important case study - it has a story to tell. It can tell us a great deal about the probable future of integrated, distributed system and network management, if only we're willing to listen.

\section{False assumptions}

To a significant degree, the failure of the OSF to deliver DME reflects false assumptions and internal contradictions underlying the DME, in the author's opinion. A few examples should suffice:

- There is no fundamental difference between network management and system management.

- Distributed management of objects is the same as management of distributed objects.

- An object-oriented system so simplifies software development that every problem is easy to solve.

- If one Management Information Model is good, then two must be even better, and three, better still!

- A production system is the merely the last release of a research prototype.

The balance of this paper explores these assumptions, one by one.

\section{Conflicting paradigms of management}

“...a paradigm is a 'reality model' in which I express my thoughts in language which reflects the semantics of my reality. Choose a different paradigm, and you will be using different semantics in your language, and be operating in a different reality... 
...[Some people] live in the Computing Paradigm. [They] solve networking problems by making them go away; those are somebody else's problems. [They] spend lots of time talking about APIs as though APIs solve communication network problems...

Networkers... live in the Network Paradigm. They assume that they own it all, ... or that what they don't own is out of scope.

Internauts live in the Internetworking Paradigm; knowing, designing, and building networks of networks where one must always assume that the other end of any connection/association will be owned and controlled by someone else."

Einar Stefferud, "Paradigms Lost"

As Einar Stefferud has pointed out, it may not be obvious that people are arriving at radically different conclusions because they are starting from radically different premises [STEF94]. The Computing Paradigm focuses on APIs, the Network Paradigm on private, closed protocols, and the Internetworking Paradigm on open protocols as a means of achieving interoperability. To a significant degree, the OSF confused itself by never consciously recognizing the internal inconsistencies among these paradigms, nor consciously choosing the most appropriate paradigm for the task at hand.

The OSF operated primarily in the Computing Paradigm, and secondarily in the Network Paradigm. This left them at a serious disadvantage in comparison to their most capable competitors, who operated for the most part in the Internetworking Paradigm. The OSF was primarily oriented toward system management rather than network management. In consequence, they focused on management APIs, such as the X/Open Management Protocol (XMP) - which, in fact, is not a protocol at all. They also placed a great deal of emphasis on the use of OSF Distributed Computing Environment (DCE) Remote Procedure Calls.

Figure 1, which follows, may help to clarify the implications of the Computing Paradigm for the DME. The central DME "cloud" represents a number of DME-capable workstations. They interact with one another by means of OSF DCE RPC. They communicate with external communication devices - such as routers and bridges - by means of the public, standard protocols, SNMP and CMIP.

In the context of the DME, DCE RPC must be viewed as an internal proprietary distribution mechanism, not as an open protocol for communication among heterogeneous systems. The OSF chose not to create a lightweight, agent-only version of the DME; therefore, there was no practical possibility that vendors of communication devices such as bridges and routers would implement DME. This, in turn, guaranteed that DME could not use DCE RPC to communicate with any management platform other than another copy of the DME. We discuss this point further in the next section.

Open APIs and essentially closed use of RPC protocols took center stage in the design and planning of DME. This left scant room for the Internetworking Paradigm, for the use of open protocols in support of heterogeneity. Open protocols were relegated to a relatively minor role - the Network Management Option - on the periphery of the DME.

There is a subtle point here, one that bears repeating. The fundamental architecture of the OSF DME was felt by OSF to support heterogeneity, in that it supported hardware and software platforms from multiple vendors; however, it was perceived by many in industry as being restricted to homogeneity, in the sense that it supported communications only with other realizations of itself, the OSF DME. Moreover, DME was realized in a way that made it unsuitable for implementation into communications gear. This resulted in a deep and fundamental schism between classical network management and DME-based systems 
management - a schism that ran directly counter to OSF's stated intent of unification of systems management and network management. The next section of this paper further elaborates on this theme.

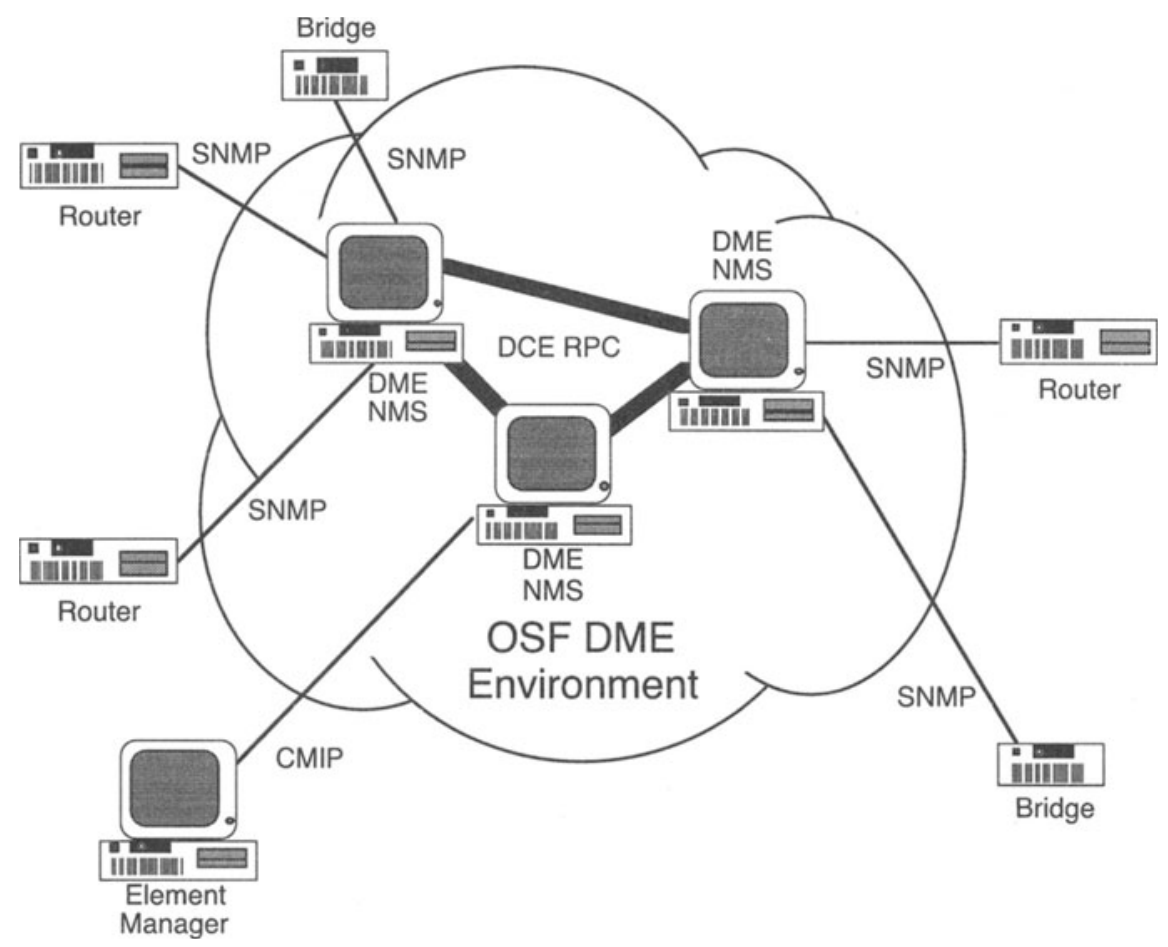

Figure 1. Management protocol environment of the OSF DME.

\section{Distributed management of objects, or management of distributed objects?}

"You might as well say," added the Dormouse, which seemed to be talking in its sleep, "that 'I breathe when I sleep' is the same thing as 'I sleep when I breathe'!"

"It is the same thing with you," said the Hatter.

Charles Lutwidge Dodgson (Lewis Carroll), Alice in Wonderland 
The DME was intended from the first to support distributed management. But... eh... what exactly was supposed to be distributed? The platform, or the things it was managing? Were they different?

The very ubiquity that was sought for the DME served in many instances to cloud the issue. DME was expected to be present on workstations from a very wide variety of workstation vendors. If DME were truly to be everywhere, then what need to distinguish between the manager and the agent -- between the ruler and the ruled? DME systems distributed everywhere could communicate happily and simply using peer protocols, in the form OSF's Distributed Communications Environment (DCE).

The reality is, of course, that we live in a pluralistic and heterogeneous world. Even in the most optimistic scenario, the DME would need to coexist with many other management solutions and technologies, just as it would need to coexist with routers and bridges in the scenario presented in the previous section. OSF's difficulty in recognizing these realities flowed naturally from the paradigms within which they operated.

The DME designers attempted to use DCE RPC and its associated mechanisms to solve a myriad of problems for the DME, from distribution to naming to security. This was a natural enough choice from point of view of engineering economy, but it severely limited the potential ability of the DME to interact with other management systems.

Consider the distribution mechanisms, for example. The OSF DCE protocols are documented, but OSF never had any concept of standardizing DME's use of DCE RPC in order to establish these protocol operations as an open protocol interface to other platforms. In consequence, DME's use of DCE had to be viewed as a closed and private mechanism for internal distribution of the DME management platform, rather than an open strategy for distributed management. It operated only within the "cloud".

Analogous inconsistencies appear in the security strategy for the DME. DME was intended to capitalize on the Kerberos-based security framework of DCE RPC, in order to achieve authentication and access control. However, this security strategy could only be relevant between copies of the DME -- elsewhere, a different strategy would have to be used for authentication and access control.

Outside of the DME distributed application, DME could presumably interact with other systems using SNMP or CMIP management protocols. The harmonization of DME's DCE-based security model, however, with those inherent in the new security features in SNMP Version 2.0 and in the GULS-based security (a generic upper-layer OSI security model) emerging for CMIP is a profoundly difficult problem. In each case, the semantics of the security model for these network management protocols differ somewhat from that of Kerberos. In general, it is difficult or impossible to map from one security model to another without losing the ability to verify the correct operation of the system.

In sum, the DME security model, and many other aspects of DME operation, are inherently applicable only to a homogeneous DME environment. They are not open, general solutions.

\section{Object orientation as a panacea}

There are many who would argue that object orientation is without a doubt the answer. I, for one, would like to better understand the question.

Object oriented programming is a very promising technique for the development of management applications. Object orientation appears to be a natural model for objects under management. Nonetheless, experience to date with true object-oriented management is somewhat limited. 
The management framework used for SNMP, the most popular management protocol, can not be said to be object-oriented. The Management Information Model of OSI network management uses object-oriented modeling techniques [OSIMIM], but not all management platforms that implement OSI network management use object-oriented techniques. Overall, the existing network management platforms can not be said to represent a compelling proof of the practicality of object-oriented management.

The ability of object-oriented solutions to scale to very large networks, to continue to operate in the face of network outages and partitions, and to accommodate changes and enhancements over time to MIBs have not been demonstrated to the author's satisfaction. It seems premature to assume that object-oriented management platforms will painlessly solve any conceivable problem.

In retrospect, it seems clear that OSF moved too quickly to embrace the Common Object Request Broker Architecture (CORBA) [CORBA] as a panacea. CORBA did not provide a defined means of interoperability among systems, nor were its interactions with traditional network management protocols specified. Immature and unstable CORBA specifications appear to have significantly delayed OSF's delivery of the Object Management Framework (OMF), the heart of the DME.

\title{
6 The Management Information Model
}

\author{
"When $I$ use a word," Humpty Dumpty said, in rather a scornful \\ tone, "it means just what I choose it to mean -- neither more nor \\ less." \\ "The question is," said Alice, "whether you can make words \\ mean so many different things." \\ Charles Lutwidge Dodgson (Lewis Carroll), \\ Through the Looking-Glass
}

The recent work of the Internet Interoperable Management Committee (IIMC), sponsored by the Network Management Forum (NMF), has demonstrated that mapping from SNMP protocols to CMIP, or vice versa, is workable ([IIMC1] and [IIMC2]). Actually, many vendors have implemented similar mappings between SNMP and CMIP over the years.

As the number of models of management information increase, however, the combinatorial problem of mapping from one to another becomes less and less tractable.

The DME supports at least three management information models:

1. The SNMP Structure of Management Information (SMI), based on RFCs 1155 [RFC1155] and 1212 [RFC1212], expressed in Concise MIB Definition notation (with a variant required in the near future, in the form of the SNMP Version 2.0 SMI [RFC1442]);

2. the OSI CMIP Management Information Model (MIM), based on ISO IS 10165-1 [OSIMIM] and -2 (the DMI) [OSIDMI], expressed in accordance with the Guidelines for the Definition of Managed Objects (GDMO, ISO 10165-4) [OSIGDMO]; and

3. a CORBA-based DME object model, expressed in I4DL.

The multiple object models confused and complicated many aspects of the DME. Consider, for instance, the DME Graphical User Interface (GUI). The DME GUI was initially tied to the 
Tivoli-derived Object Management Framework (OMF) of the DME. The GUI could not act on the attributes of SNMP or CMIP objects in the absence of an adapter object that would map the object to an equivalent I4DL definition. Initially, OSF did not intend to provide any adapter objects. This would have resulted in a GUI with no cognizance at all of SNMP objects! OSF, under prodding from its user group, implemented an adapter object for SNMP MIB-I.

Mapping among these three management information models could be cumbersome or even impractical. There are two possibilities: provide any-to-any translation, or translate everything into a common model (which, in the case of the DME, would have to be I4DL).

Consider an example. Suppose that you speak German, and I speak English. We can solve our communication problems by learning one another's language, and learning to translate from one to the other. This is the IIMC approach. Granted, there may be some loss of meaning when going from a language with richer semantics to a language with more meager semantics, but we should still be able to communicate after a fashion. This works well enough.

Add a French speaker to our little clique. We now have three possible translations. Add a Spaniard, and we have six. It quickly gets out of hand.

An alternative approach is for both of us to agree to speak some third language -- an Esperanto. This avoids the proliferation of required translations. If we are only trying to translate between German and English, however, this is less satisfactory than direct translation, because the likelihood of loss of meaning is far greater when we translate everything twice. The Esperanto approach becomes more attractive if everyone can be induced to use it, yet one has to wonder: Esperanto could be viewed as a technical success, but a marketing failure. Does I4DL really make sense as the lingua franca of the DME?

Moreover, the translation of SNMP MIB definitions of GDMO MO specifications into CORBA IDL must still be viewed today as a research project, not as a trivial matter of software engineering. The Joint Inter-Domain Task Force (JIDM) of X/Open and the NM Forum has made a good start in this area ([JIDM1] and [JIDM2]), but it is only a start.

The OSF developed a methodology for translating SNMP MIBs into I4DL. They considered serveral possible approaches to this translation. One possibility was to perform a very direct and literal translation of the SNMP definition; a second was to create a highly abstract and useroriented I4DL specification based on the SNMP MIB definitions; and a third was to create a definition that follwed the original SNMP MIB closely, but with some adaptations in the interest of neatness and comprehensibility. After some thought, they settled on the last of these three options, and translated SNMP MIB I as a proof of concept.

The problem with this third approach is that it implies manual translation of SNMP MIBs, rather than fully automated translation. This is impractical. New SNMP MIBs are proliferating rapidly today. A typical SNMP management product simply compiles the SNMP MIB definitions in order to provide at least a primitive access to management information - neither software development nor human intelligence are required, ideally, in order to access a new device with a new MIB. In the absence of an automated translation capability, OSF could have no hope of catching up with the flood of new MIB definitions.

The NMF-sponsored IIMC project, by contrast, chose a mapping from SNMP MIBs into GDMO that is largely mechanical. It can be done. In fact, an object-oriented infrastructure like that of the DME lends itself to the most primitive possible translation from external managament information models to internal ones - more complex and user-friendly views can always be layered on top of simple and direct translations. 


\section{Software integration}

"Why should we be in such desperate haste to succeed, and in such desperate enterprises?"

Henry David Thoreau, Walden

Back in September of 1991, when the OSF first announced the technologies it had selected from those submitted in response to the DME RFT (Request For Technology), many knowledgeable observers concluded that the OSF had bitten off more than it could chew. OSF's initial strategy was to merge HP OpenView's SNMP and CMIP protocol implementations with Bull's XMP API software, to integrate the Wang / Banyan event services in with these, and to overlay all of these with Tivoli's object-oriented management platform and IBM's data engine, as depicted in Figure 2 below [OSF91]. This management framework would then be integrated with a variety of management applications, which would serve to demonstrate the capabilities of the management framework.

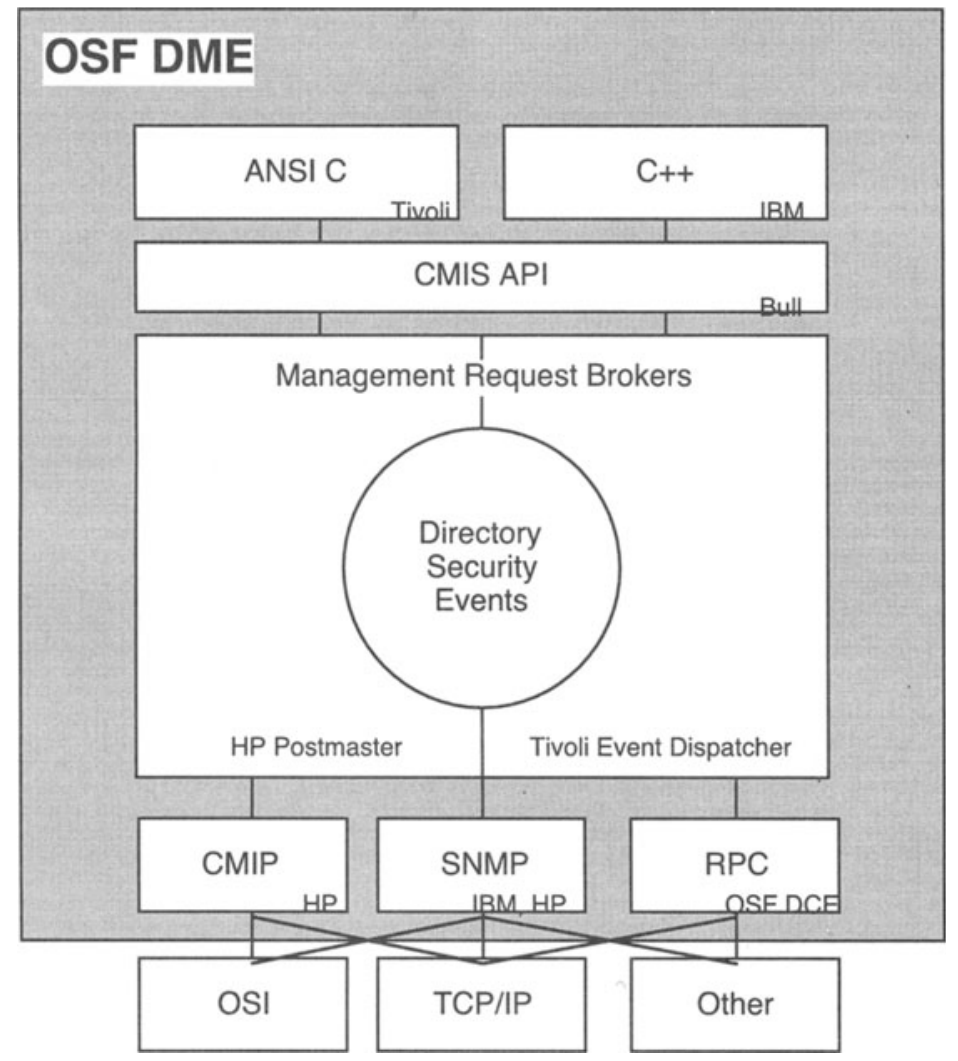

Figure 2. Implementation architecture of the OSF DME. 
The notion that a single coherent system could quickly be created from these widely disparate piece parts was simply naive. Furthermore, the software components were not mature enough to be used as OSF intended.

It should also be clear from the foregoing discussion that many aspects of the DME involved advanced technologies that were barely beyond the stage of research. They were not yet ripe for production deployment.

\section{Concluding remarks}

"Those of us who succeed, and fail to push on to a still greater failure, are the true spiritual middle-classers."

Eugene O’Neill

Clearly the OSF did not realize their original DME objectives. Were they foolish to have tried?

In the author's opinion, they were not. OSF realized at the outset that the world of the Nineties did not need the OSF to create yet another SNMP MIB browser. To do so would intrude on existing products that were already successful in the marketplace, without offering software of greater utility to end users.

Instead, OSF attempted to leapfrog the existing state of the art. This may have been a risky approach, but it is not clear, from a business perspective, that they had better alternatives. More to the point, the problem that the OSF was trying to solve was the right problem. The integration of systems management and network management may be as elusive today as it was in 1990, but potentially it is just as valuable today as it appeared to be in 1990 .

We should all strive to keep the lessons of the DME in mind as we move forward with other management projects. The underlying technology was subtly complex. It was not yet ready for "prime time". The most fundamental shortcoming of the DME is that it over-reached itself it flew too high, too far above the comfortable and the mundane, too close to the sun.

At the same time, we should strive to remember that Icaros did not fly alone. His father, the master technologist Daedalus, learned from his son's mistakes and ultimately succeeded where Icaros failed. If we wish to ultimately rise above the limitations of today's management systems, we must be willing not only to take risks, but also to learn from the errors of others.

\section{References}

[OSFRFT] Open Software Foundation, "Request for Technology: Distributed Management Environment", August, 1990.

[OSF91] Open Software Foundation, "OSF Distributed Management Environment Rationale", September, 1991.

[OSF94] Open Software Foundation, "Distributed Management Environment: An Overview", March, 1994.

Open Software Foundation, "Using DCE and DME to Manage Software in DCE-Based Environments", March, 1994. 
Open Software Foundation, “OSF Distributed Management Environment: The DME Network Management Option" (brochure), April, 1994.

Bruce Papazian and J. Scott Marcus, "Issues for a Graphical User Interface for the DME RFT", June, 1991.

W.H.D. Rouse, Gods, Heroes and Men of Ancient Greece: Mythology's Great Tales of Valor and Romance, Mentor, 1957.

[STEF94] Einar Stefferud, "Paradigms Lost", in ConneXions, Volume 8, Number 1, January, 1994.

Charles Lutwidge Dodgson (Lewis Carroll), Alice's Adventures in Wonderland, Random House, 1946. Originally published in Great Britain in 1865.

Charles Lutwidge Dodgson (Lewis Carroll), Through the Looking-Glass, Random House, 1946. Originally published in Great Britain in 1872.

[RFC1155] RFC 1155, M. Rose and K. McCloghrie, "Structure and Identification of Management Information for TCP/IP based internets", May 1990.

[RFC1212] RFC 1212, M. Rose, K. McCloghrie - Editors, "Concise MIB Definitions", March 1991.

[RFC1442] RFC 1442, PS, J. Case, K. McCloghrie, M. Rose, S. Waldbusser, "Structure of Management Information for version 2 of the Simple Network Management Protocol (SNMPv2)", May, 1993.

[OSIMIM] ISO/IEC 10165-1, Information Technology - Open Systems Interconnection Structure of Management Information - Part 1: Management Information Model, 1991.

[OSIDMI] ISO/IEC 10165-2, Information Technology - Open Systems Interconnection Structure of Management Information - Part 2: Definition of Management Information, 1992.

[OSIGDMO] ISO/IEC 10165-4, Information Technology - Open Systems Interconnection Structure of Management Information - Part 4: Guidelines for the Definition of Managed Objects, 1991.

[IIMC1] ISO/CCITT and Internet Management Coexistence (IIMC): Translation of Internet MIBs to ISO/CCITT GDMO MIBs, Draft 3, August 1993.

ISO/CCITT and Internet Management Coexistence (IIMC): ISO/CCITT to Internet Management Proxy, Draft 3, August 1993.

ISO/CCITT and Internet Management Coexistence (IIMC): ISO/CCITT to Internet Management Security, Draft 3, August 1993.

[IIMC2] ISO/CCITT and Internet Management Coexistence (IIMC): Translation of ISO/CCITT GDMO MIBs to Internet MIBs, Draft 3, August 1993.

[CORBA] Object Management Group, The Common Object Request Broker: Architecture and Specification, OMG Document Number 91.12.1, December, 1991.

[JIDM1] Subrata Mazumdar, "Translation of SNMPv2 MIB Specification into CORBA-IDL: A Report of the Joint Xopen/NM Forum Inter-Domain Taskforce", July, 1993.

[JIDM2] Tom Rutt, "Comparison of the OSI management, OMG and Internet management Object Models: A Report of the Joint XOpen/NM Forum Inter-Domain Management Task force," March, 1994. 